\title{
A Frequency Domain Direct Localization Method Based on Distributed Antenna Sensing
}

\author{
Gaofeng Zhao $\mathbb{D}^{1,2}$ Hao Zheng $\mathbb{D}^{1},{ }^{1}$ Yingying $L i \mathbb{D}^{1,2}$ Kehui Zhu $\mathbb{D}^{1,2}$ and Jianfeng $\operatorname{Li} \mathbb{D}^{1,2}$ \\ ${ }^{1}$ College of Electronic Information Engineering, Nanjing University of Aeronautics and Astronautics, Nanjing 211106, China \\ ${ }^{2}$ Key Laboratory of Dynamic Cognitive System of Electromagnetic Spectrum Space (Nanjing University of Aeronautics \\ and Astronautics), Ministry of Industry and Information Technology, Nanjing 211106, China
}

Correspondence should be addressed to Gaofeng Zhao; zhaogaofeng@nuaa.edu.cn and Jianfeng Li; lijianfeng@nuaa.edu.cn

Received 22 December 2020; Revised 26 April 2021; Accepted 19 May 2021; Published 2 June 2021

Academic Editor: Weizhi Meng

Copyright ( 2021 Gaofeng Zhao et al. This is an open access article distributed under the Creative Commons Attribution License, which permits unrestricted use, distribution, and reproduction in any medium, provided the original work is properly cited.

Traditional two-step passive localization methods need to extract the parameters like the direction of arrival (DOA), time of arrival (TOA), and time difference of arrival (TDOA) from the original data to determine the source position, which causes the poor positioning accuracy due to error accumulation. In this paper, a direct position determination (DPD) method is proposed to improve the positioning accuracy and robustness, which is based on a correlation algorithm. Firstly, the cost function directly related to the location of the source can be established by synthesizing the data received by multiantenna in the frequency domain. Then, the position of the source is estimated by the correlation DPD method to search the monitoring area. Compared to the improved TDOA algorithm and Least Squares DPD algorithm, the proposed method shows better localization accuracy of different SNRs. Finally, based on real measured data, it can be seen that the results of the proposed algorithm are better than the improved TDOA algorithm.

\section{Introduction}

Wireless positioning is an important research direction in the civilian and military fields [1]. There are many wireless positioning algorithms, among which the two-step positioning algorithms are the most concerned. The traditional twostep positioning methods extract measurement parameters such as angles of arrival (AOA), times of arrival (TOA), and time differences of arrival (TDOA) from the received signal, then solve the equations established based on extracted measurement parameters to obtain the target position [2, 3]. In order to improve the positioning accuracy, a TDOA positioning method based on time delay compensation was proposed in [4], which improved the performance of twostep positioning to a certain extent. In [5], considering the synchronization error between transmitter and receiver, a passive location method of the asynchronous receiver based on expectation maximization is proposed. The method significantly improves the positioning performance. In [6], a two-dimensional direction of arrival (DOA) and polarization estimation algorithm for noncircular signals based on multi- signal classification (MUSIC) is proposed. The algorithm is mainly applied to three-dimensional millimeter-wave polarized massive multi-input multioutput (MIMO) systems. In [7], a novel system architecture including a reconfigurable intelligent surface (RIS) and multiple autonomous vehicles or a massive multi-input multioutput (MIMO) is considered in vehicle location systems. However, the losses of position information and errors cannot be avoided due to the separation of the parameter solution and target localization for twostep localization methods. Besides, the measurement parameters may not match the corresponding transmitter, and the positioning performance is poor with low SNR $[8,9]$. Therefore, it has become an important research topic in the field of wireless positioning to find a method to further improve the positioning accuracy of the target signal source.

In [10], a direct location algorithm (Least Squares DPD) based on angle and time delay information is proposed, which firstly proved that the direct positioning accuracy is better than the two-step positioning accuracy. In [11], a multisource direct location method without knowing the number of transmitters is proposed. Different from the traditional 
two-step positioning, the direct location technology can directly estimate the emitter position without parameter estimation by using the received signal [12]. It avoids the information loss caused by the two-step positioning method and obtains higher positioning accuracy. Besides, the direct location algorithm solves the parameter matching problem in the traditional two-step localization method in the case of multiple sources [13]. Although direct position determination (DPD) does not need to estimate the parameters, we still need to consider which variables are contained in the emitter location information and select the appropriate information type according to the location scene. A direct location method based on time difference of arrival (TDOA) and frequency difference of arrival (FDOA) was proposed in [14-16] by using multiple moving single sensors to intercept the signal of the stationary emitter. Considering the information of TDOA and FDOA, the model between the intercepted signal and the emitter location was established directly, and the direct location was realized by the exhaustive search method. In [17], a multistation direct location method based on TDOA was proposed on the condition that the observation stations and emitters are relatively stationary. A new direct location method using a multiple antenna array (DPD) algorithm was proposed in [18]. This method uses the cross-correlation matrix (CCM) of the received signals to estimate the position of the emitter directly. An algorithm for DPD of multiple fixed transmitters was shown in [19] by using the position information embedded in the angle and Doppler frequency shift. In [20], a direct position estimation (DPE) algorithm for dense multipath channels based on Gaussian approximation is proposed for passive location in a multipath environment. In [21], the influence of Doppler frequency shift on direct location is considered and describes the fluctuation of DPD cost function in NLOS. In [22], the problem of determining the unknown location of a mobile station in a millimeter-wave MIMO system is solved. The exact solution of the maximum likelihood estimation problem is provided, and the basic lower bounds of channel and location parameter estimation uncertainty are derived. In [23], the maximum likelihood (ML) direct position estimation (DPE) problem of the multiantenna receiver in a dynamic multipath environment is studied, and a complexity reduction algorithm based on the pseudo ML method is proposed.

With the development of radar and communication technology, the modulated broadband signal has been more and more widely used for its advantages of a large amount of information and strong antijamming ability. However, there are few direct positioning algorithms for broadband signals. In [24], the direct location of wideband signals was firstly considered, and a model for intercepting wideband signals by moving multistations was established. Besides, a direct location cost function based on TDOA and FDOA was established by expanding the application of direct location to broadband signals. In [25], a maximum likelihood direct location method based on TDOA was proposed, which is aimed at the case of an unknown signal. Based on the continuous signal model, the method can effectively locate the wideband or narrowband signal target. In [26], the coherent and incoherent direct localization methods are studied for wideband signals. By using the two preprocessing techniques, broadband processing gain can be improved.

In the positioning scene of distributed multiantenna system, a direct positioning method based on the improved MUSIC algorithm is proposed in [27] to solve the joint detection and positioning problem of main transmitters in a cognitive radio network. In [28], based on simulations by applying a distributed antenna system for localization purposes in indoor scenarios, an extensive study was presented. According to [29], based on the perspective of information theory, in the absence of GPS, wireless local area network (WLAN) positioning technology is recognized as one of the preferred positioning technologies. In [30], a novel system architecture was constructed, which is consisting of multiple noncooperative unmanned aerial vehicles (UAVs) and the software-defined Internet of vehicles (SDN-IoV). And two source enumeration methods are proposed in a complex SDN-IoV environment with color noise to locate the positions of vehicles and pedestrians.

In this paper, we propose a frequency domain direct position determination method to improve the location accuracy of emitters in distributed multiantenna scenarios. The data receiving model under distributed nodes is established, and the data of each node is fused. The data model after fusion is obtained by accumulating frequency points. Based on the phase information, the cost function is constructed, and the location of the emitter is determined by grid searching in the monitored area. Finally, we examine the performance of the proposed algorithm from two aspects. Through simulations, the algorithm is compared with Least Squares DPD algorithm in [10], DPD EIGEN algorithm in [11], and TDOA improved algorithm in [4]. Then, the performance of the proposed algorithm is further verified by processing the real measured data.

The contributions of this paper are summarized as follow:

(1) In this paper, a distributed multiantenna direct location method is proposed. At present, most direct localization methods are mainly aimed at narrowband signals. The method proposed in this paper can process both narrowband and wideband signals utilizing multifrequency points accumulation

(2) The proposed algorithm was compared with the existing algorithms by simulation, including the TDOA improved algorithm in [4], the LS DPD algorithm in [10], and the DPD EIGEN algorithm in [11]. The results show that the performance of the proposed algorithm has been improved

(3) The proposed algorithm is used to process the measured data, and the result of the target location is obviously better than that of the TDOA improved algorithm

\section{Signal Model}

The system model is shown in Figure 1, where an unknown signal source exists in position $p(x, y)$. There are $L(L \geq 4)$ monitoring stations in the spatial domain that can receive 


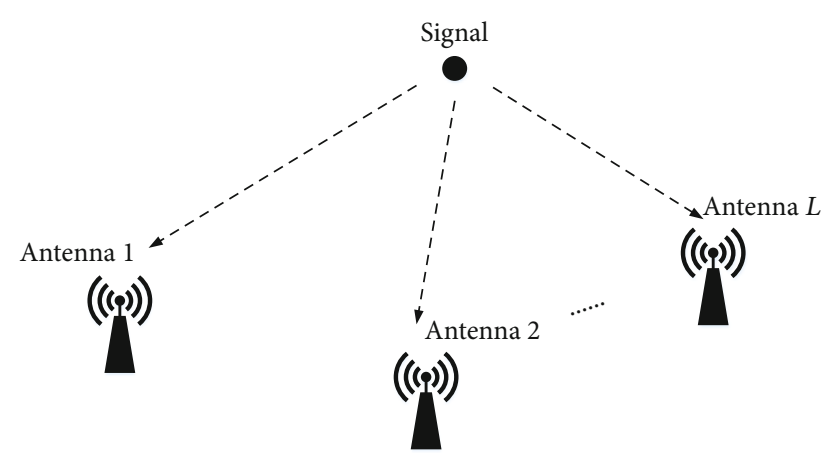

FIgURE 1: Multiantenna positioning scene diagram.

signals, and the positions of each base station are denoted as $B_{1}\left(x_{1}, y_{1}\right), \cdots, B_{L}\left(x_{L}, y_{L}\right)$. The signal observed by the $l$-th $(1 \leq l \leq L)$ base station antenna is given by

$$
y_{l}(t)=\alpha_{l} s\left(t-\tau_{l}-t_{0}\right)+n_{l}(t), 1 \leq l \leq L,
$$

where $\alpha_{l}$ is an unknown complex scalar representing channel attenuation. $s\left(t-\tau_{l}-t_{0}\right)$ is the signal waveform, transmitted at a time $t_{0}$ and delayed by $\tau_{l} . n_{l}(t)$ represents noise. The sampled version of the signal in (1) is given by

$$
\begin{aligned}
& y_{l}(k)=\alpha_{l} s(k)+n_{l}(k), \quad 0 \leq k \leq K-1, \\
& \left.s_{l}(k) \triangleq s\left(t-\tau_{l}-t_{0}\right)\right|_{t=k T}, \\
& \left.y_{l}(k) \triangleq y_{l}(t)\right|_{t=k T}, \\
& \left.n_{l}(k) \triangleq n_{l}(t)\right|_{t=k T},
\end{aligned}
$$

where the sampling interval is $T$ and the discrete expressions of signals received by each base station are

$$
\begin{gathered}
y_{1}(k)=\alpha_{1} s_{1}(k)+n_{1}(k), \quad 0 \leq k \leq K-1, \\
\vdots \\
y_{L}(k)=\alpha_{L} s_{L}(k)+n_{L}(k), \quad 0 \leq k \leq K-1 .
\end{gathered}
$$

\section{Improved TDOA Positioning Method}

According to the received signals of each antenna in (1), the traditional TDOA method is used to locate the emitter. Taking the signal received by the first base station as the reference signal, the generalized cross-correlation (GCC) algorithm is used to obtain the delay difference $\tau_{i 1}, i=2, \cdots, L$ of the received signals between other base stations and the reference base station.

However, in engineering, $\tau_{i 1}, i=2, \cdots, L$ is usually not an integral multiple of the sampling interval. Due to the limitation of the sampling frequency, there is always a noninteger delay between the most accurate delay difference that can be distinguished by digital sampling and the true time delay difference.

To solve this problem, a time delay estimation method based on time delay compensation is proposed in [4]. The main steps of this algorithm are as follows: firstly, a reference signal is selected, and a small delay unit is defined to compen- sate for the delay of other signals. Then, the delay compensated signal is cross-correlated with the reference signal. Finally, a high precision delay estimation is obtained based on the optimal cross-correlation results.

After obtaining the delay difference of each base station relative to the reference base station, the target position can be determined by solving the Chan function. The distance between the base station and the signal source is as follows:

$$
R_{i}=\sqrt{\left(x_{i}-x\right)^{2}+\left(y_{i}-y\right)^{2}}, i=1, \cdots, L .
$$

When $L=3$, there are three base stations participating in positioning, and the position of the radiation source can be obtained according to the Chan algorithm:

$$
[x y]=\left[\begin{array}{ll}
x_{2,1} & y_{2,1} \\
x_{3,1} & y_{3,1}
\end{array}\right]^{-1} \times\left\{\left[\begin{array}{l}
R_{2,1} \\
R_{3,1}
\end{array}\right] * R_{1}+\frac{1}{2}\left[\begin{array}{l}
R_{2,1}^{2}-K_{2}+K_{1} \\
R_{3,1}^{2}-K_{3}+K_{1}
\end{array}\right]\right\},
$$

where $K_{i}=x_{i}^{2}+y_{i}^{2}, i=1,2,3, x_{\mu, 1}$, and $y_{\mu, 1}$ represent the difference between the coordinates of the base station,

$$
\begin{aligned}
x_{\mu, 1} & =x_{\mu}-x_{1}, \\
y_{\mu, 1} & =y_{\mu}-y_{1}, \\
R_{\mu, 1} & =R_{\mu}-R_{1}, \mu=2, \cdots, L,
\end{aligned}
$$

where $R_{\mu, 1}=\sqrt{\left(x_{\mu}-x\right)^{2}+\left(y_{\mu}-y\right)^{2}}-\sqrt{\left(x_{1}-x\right)^{2}+\left(y_{1}-y\right)^{2}}$.

\section{DPD Algorithm}

By performing DFT on (2), we can get

$$
\begin{aligned}
& Y_{1}\left(w_{k}\right)=\alpha_{1} S\left(w_{k}\right) e^{-j w_{k} \tau_{1}}+N_{1}\left(w_{k}\right), \\
& \vdots \\
& Y_{L}\left(w_{k}\right)=\alpha_{L} S\left(w_{k}\right) e^{-j w_{k} \tau_{L}}+N_{L}\left(w_{k}\right), \\
& 0 \leq k \leq K-1 ; w_{k} \triangleq \frac{2 \pi k}{K T},
\end{aligned}
$$

where $Y_{l}\left(w_{k}\right)$ is the frequency domain form of the signal received by the $l$-th base station, $S\left(w_{k}\right)$ represents the discrete Fourier transform of the signal $s(k)$, and $N_{1}\left(w_{k}\right)$ represents the Fourier transform of the noise corresponding to the $l$-th base station.

4.1. Traditional DPD Algorithm. According to the frequency domain signal expression of Equation (8), the cost function related to the location of the source is established. By minimizing the cost function related to the location of the source, the least square estimation result of the location of the emitter can be obtained

$$
Q(p)=\sum_{l=1}^{L} \sum_{k=0}^{K-1}\left\|Y_{l}\left(w_{k}\right)-\left(\alpha_{l} S\left(w_{k}\right) e^{-j w_{k} \tau_{l}}\right)\right\|^{2},
$$


where $\|\cdot\|$ represents norm operation and the cost function is expressed as the sum of the following items:

$$
\begin{aligned}
Q(p) & =\sum_{l=1}^{L} Q_{l}(p), \\
Q_{l}(p) & \triangleq \sum_{k=1}^{K}\left\|Y_{l}\left(w_{k}\right)-\left(\alpha_{l} S\left(w_{k}\right) e^{-j w_{k} \tau_{l}}\right)\right\|^{2} \\
& \triangleq \sum_{k=1}^{K}\left\|Y_{l}\left(w_{k}\right)-\left(\alpha_{l} b_{l}(p)\right)\right\|^{2},
\end{aligned}
$$

where $b_{l}(p)=S\left(w_{k}\right) e^{-j w_{k} \tau_{l}}$, in order to minimize the cost function, the attenuation coefficient $\alpha_{l, m}$ in (10) is estimated and the result is shown in (11).

$$
\widehat{\alpha}_{l}=\left(\left(b_{l}(p)\right)^{H} b_{l}(p)\right)^{-1}\left(b_{l}(p)\right)^{H} Y_{l}\left(w_{k}\right)
$$

For the sake of generality, assume the following

$$
\left\|b_{l}(p)\right\|^{2}=1 \quad \forall l
$$

Finally, the emitter location is determined by solving the matrix eigenvalue containing the emitter location information.

4.2. The Proposed DPD Algorithm. The delay is related to the signal position $p(x, y)$. Suppose the propagation velocity of the electromagnetic wave is $c$, the expression of $\tau_{l}(p)$ is (13), which is related to $p(x, y)$.

$$
\begin{gathered}
\tau_{l}=\frac{\sqrt{\left(x-x_{l}\right)^{2}+\left(y-y_{l}\right)^{2}}}{c} . \\
\tau_{l}(p) \triangleq \tau_{l}
\end{gathered}
$$

According to the frequency domain signal expression obtained in (8), all the monitoring data are synthesized

$$
\begin{aligned}
\mathbf{Y}\left(w_{k}\right) & =\left[\begin{array}{c}
Y_{1}\left(w_{k}\right) \\
Y_{2}\left(w_{k}\right) \\
\vdots \\
Y_{L}\left(w_{k}\right)
\end{array}\right]=\left[\begin{array}{c}
e^{-j w_{k} \tau_{1}(p)} \alpha_{1} \\
e^{-j w_{k} \tau_{2}(p)} \alpha_{2} \\
\vdots \\
e^{-j w_{k} \tau_{L}(p)} \alpha_{L}
\end{array}\right] S\left(w_{k}\right)+\left[\begin{array}{c}
N_{1}\left(w_{k}\right) \\
N_{2}\left(w_{k}\right) \\
\vdots \\
N_{L}\left(w_{k}\right)
\end{array}\right] \\
= & \boldsymbol{\Phi}(\alpha) \mathbf{a}\left(w_{k}, p\right) S\left(w_{k}\right)+\mathbf{N}\left(w_{k}\right),
\end{aligned}
$$

where $\boldsymbol{\Phi}(\alpha)=\operatorname{diag}\left(\alpha_{1}, \cdots, \alpha_{L}\right) \in \mathbb{C}^{L \times L}$ is a diagonal matrix containing all attenuation coefficients. $\mathbf{a}\left(w_{k}, p\right) \in \mathbb{C}^{L \times 1}$ is the delay factor vector corresponding to different monitoring positions, and its expression is shown in

$$
\mathbf{a}\left(w_{k}, p\right)=\left[\begin{array}{c}
e^{-j w_{k} \tau_{1}(p)} \\
e^{-j w_{k} \tau_{2}(p)} \\
\vdots \\
e^{-j w_{k} \tau_{L}(p)}
\end{array}\right] .
$$

Then, the cost function directly related to the position $p(x, y)$ is established as

$$
\max _{p} \sum_{k=1}^{K}\left|\mathbf{Y}^{H}\left(w_{k}\right) \boldsymbol{\Phi}(\alpha) \mathbf{a}\left(w_{k}, p\right)\right|
$$

The correlation lies in the phase compensation; $\boldsymbol{\Phi}(\alpha)$ is a real number and independent of the frequency point. So the cost function can be modified as follows:

$$
\max _{p} \sum_{k=1}^{K}\left|\mathbf{Y}^{H}\left(w_{k}\right) \mathbf{a}\left(w_{k}, p\right)\right|
$$

Then, a series of spatial grid points are obtained by grid division of the monitoring area. Based on the correlation DPD method, the cost function after signal synthesis can be solved. According to (17), the grid point that meets the requirements can be obtained, which is the location of the target interference source.

The main steps of the algorithm are as follows:

Step 1. Signal input: the signal received by each node is transformed by DFT, and the frequency domain data of each node is fused to form a signal matrix $\mathbf{Y}\left(w_{k}\right) \in \mathbb{C}^{L \times 1}$ as the input of the algorithm.

Step 2. Simplified signal matrix: considering that the algorithm is only related to the phase information of the signal, the attenuation coefficient matrix in the signal matrix can be removed, and the signal matrix related to phase can be obtained by simplification.

Step 3. The phase information is used to estimate the source position: the area to be monitored is gridded. According to the cost function of (17), the most relevant grid point that is corresponding to the estimated source location can be found.

4.3. Algorithm Analysis. In this paper, the direct source location algorithm belongs to the correlation algorithm, which mainly uses the phase information in the signal. When the monitoring area is large and there are many grid areas, the delay factor vectors $\mathbf{a}\left(w_{k}, p\right)$ in (17) will generate phase ambiguity. It is assumed that there are $M$ grid points in the monitoring area, and the phases formed by two grid points $m_{1}$ and $m_{2}$ at the $l$-th base station are as follows: $\varphi_{l, m_{1}}=w_{k} \cdot \tau_{l}\left(p_{m_{1}}\right)$ and $\varphi_{l, m_{2}}=w_{k} \cdot \tau_{l}\left(p_{m_{2}}\right)$. When $\varphi_{l, m_{2}}=$ $\varphi_{l, m_{1}}+r \cdot 2 \pi,(r \neq 0, r \in Z)$, we can obtain 


$$
\begin{aligned}
e^{j \varphi_{l, m_{2}}} & =e^{j\left(\varphi_{l, m_{1}}+r \cdot 2 \pi\right)}=e^{j \varphi_{l, m_{1}} \cdot e^{j r \cdot 2 \pi}} \\
& =e^{j \varphi_{l, m_{1}}} \cdot[\cos (r \cdot 2 \pi)+j \sin (r \cdot 2 \pi)]=e^{j \varphi_{l, m_{1}}} .
\end{aligned}
$$

It can be seen from (18) that different grid points in the monitoring area may have the same delay factor for the same monitoring measurement, which will affect the positioning accuracy of relevant algorithms. The fuzzy positioning diagram is shown in Figure 2.

As can be seen from Figure 2, for a base station, the same delay factor may correspond to multiple different locations.

As shown in Figure 3, for multiple base stations, the possible fuzzy location points of each base station are different, but the real source points are the same. At the same time, the false position points of different frequency points are also different, so combining the data of multiple base stations can reduce the influence of some phase ambiguity on positioning and improve the accuracy of positioning.

4.4. Cramer-Rao Bound. The positioning performance of the algorithm will be compared with that of the CRB for the model (Equation (14)), which are comprised in the parameter vector $\rho \in \mathbb{C}^{2(K+1) \times 1}$

$$
\boldsymbol{\rho}=\left[s_{1}^{(r) T}, s_{1}^{(c) T}, \cdots, s_{K}^{(r) T}, s_{K}^{(c) T}, p^{T}\right]
$$

where $s_{k}^{(r) T}$ and $s_{k}^{(c) T}$ are the real and imaginary part of the source signals and $p^{T}=(x ; y)$ is the source position to be estimated. The CRB is given by the inverse Fisher Information Matrix (FIM), i.e.,

$$
E\left\{(\boldsymbol{\rho}-\widehat{\boldsymbol{\rho}})(\boldsymbol{\rho}-\boldsymbol{\rho} \wedge)^{T}\right\} \geq \mathbf{J}^{-1}(\boldsymbol{\rho})
$$

With

$$
\mathbf{J}(\boldsymbol{\rho})=E\left\{\left(\frac{\partial \mathscr{L}(\mathbf{Y} ; \boldsymbol{\rho})}{\partial \boldsymbol{\rho}}\right)\left(\frac{\partial \mathscr{L}(\mathbf{Y} ; \boldsymbol{\rho})}{\partial \boldsymbol{\rho}}\right)^{T}\right\}
$$

where $\mathscr{L}$ is the log-likelihood function as a function of the collection of all data $\mathbf{Y}=\left[Y_{1}, \cdots, Y_{K}\right] \in \mathbb{C}^{L \times K}$ :

$$
\mathscr{L}(\mathbf{Y} ; \boldsymbol{\rho})=-K L \ln \left(\pi \sigma_{n}^{2}\right)-\frac{1}{\sigma_{n}^{2}} \sum_{k=1}^{K}\left|Y_{k}-\mathscr{A} \mathbf{s}_{k}\right|^{2}
$$

In this log-likelihood function, $\mathscr{A}=\mathbf{a}\left(w_{k}, p\right) \in \mathbb{C}^{L \times 1}$. According to [31], the CRB is as follows:

$$
\operatorname{CRB}(p)=\frac{\sigma_{n}^{2}}{2}\left(\sum_{k=1}^{K} \operatorname{Re}\left\{S_{k}^{H} D^{H} P_{A}^{\perp} D S_{k}\right\}\right)^{-1}
$$

with $P_{\mathscr{A}}^{\perp}=\mathbf{I}_{L}-\mathscr{A}\left(\mathscr{A}^{H} \mathscr{A}\right)^{-1} \mathscr{A}^{H} \in \mathbb{C}^{L \times L}, \quad D=[\partial \mathscr{A} / \partial x, \partial \mathscr{A} / \partial y]$ $\in \mathbb{C}^{L \times 2 L}$, and $S_{k}=\mathbf{I}_{2} \otimes \mathbf{s}_{k} \in \mathbb{C}^{2 L \times 2}$.

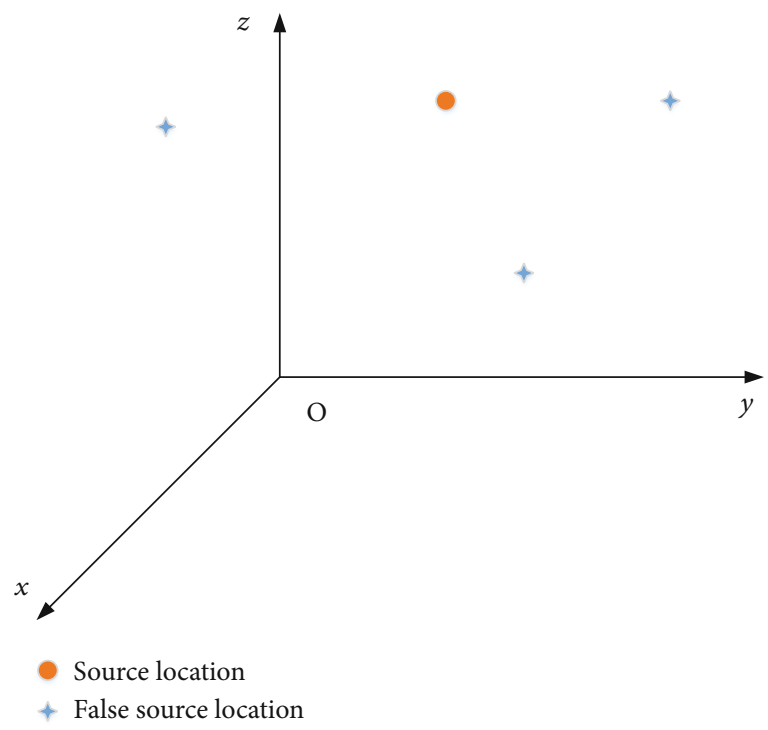

Figure 2: Fuzzy location diagram for one node.

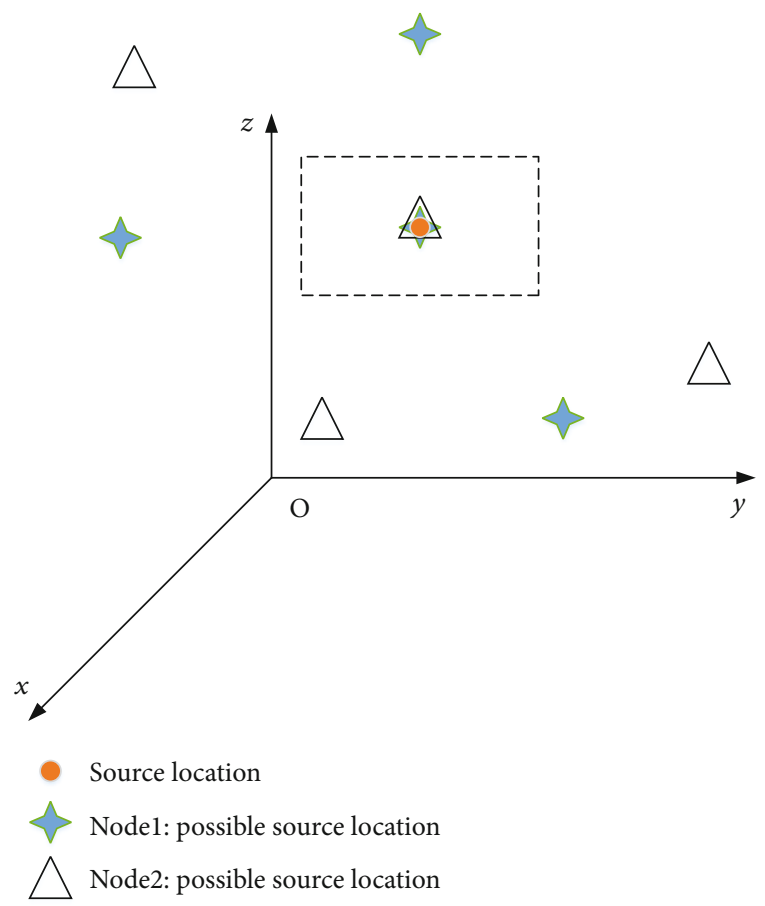

FIGURE 3: Schematic diagram of the reduced monitoring area.

4.5. Complexity Analysis. For the proposed method, its computational complexity is $O\left(L^{2} T^{2}+L K\right)$, and $T$ is the number of grids searched on coordinates $x$ and $y$. The complexity of the improved TDOA positioning method is $\mathrm{O}(L(2 J+K)+$ $\left.3(L-1) K \log _{2}^{K}+(L-1) K^{2}+7(L-1)^{2}\right)$, where $J$ is the number of cross-correlation. The complexity of LS_DPD algorithm and DPD_EIGEN algorithm is $O\left(K T^{2}+T^{4}\right)$ and $O\left(K T^{2}+6 K L^{2}+L T^{2}+K L\right)$, respectively. 


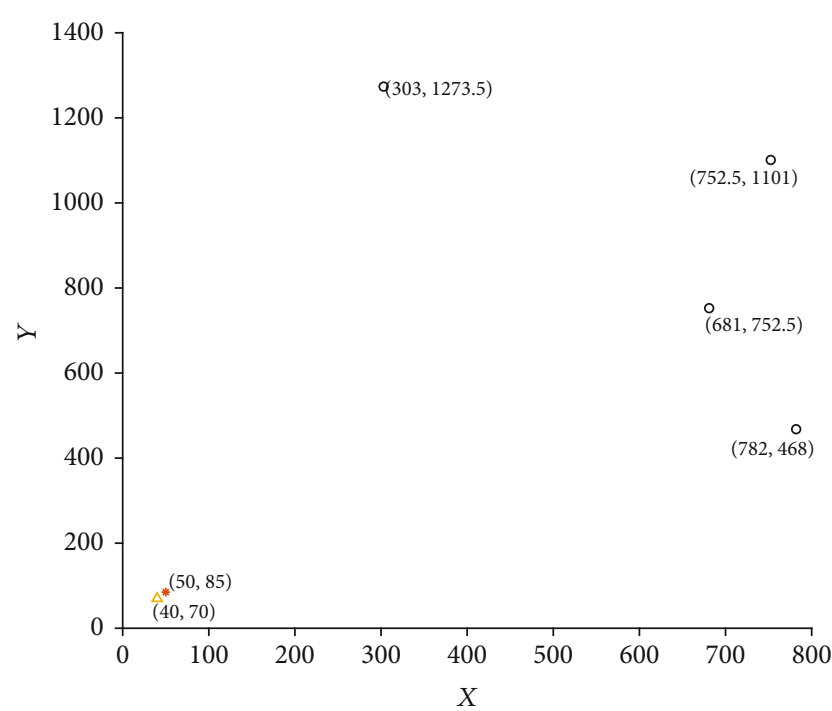

- Antenna location

* Signal location

$\triangle$ Source estimation location

FIGURE 4: Locating result with $\mathrm{SNR}=-5 \mathrm{~dB}$.

\section{Algorithm Verification}

5.1. Simulation Results. In this part, root mean square error (RMSE) is employed to verify the performance of the algorithm; the expression of it is

$$
\mathrm{RMSE}=\sqrt{\frac{1}{M} \sum_{m=1}^{M}\left\|p \wedge_{m}-p\right\|^{2}}
$$

where $M$ is the number of Monte Carlo experiments, $p$ is the true location of the unknown source, and $\widehat{p}_{m}$ is the estimation result of the source location of the $m$-th Monte Carlo experiment.

Figure 4 shows the localization performance of the algorithm without considering the multipath signal, and the SNR is set to $-5 \mathrm{~dB}$. There are four receiving base stations with coordinates of $(303,1273.5),(752.5,1101),(681,752.5$ ) , and $(782,468)$. And the coordinate of the unknown source is $(50,85)$. The simulation results show that the algorithm can effectively estimate the source location with low SNR, and the estimated source location is $(40,70)$. When considering the influence of the multipath effect, the estimated result of the source is $(88,78)$ in Figure 5.

In order to further verify the performance of the proposed algorithm, we compare the proposed algorithm with the improved TDOA algorithm, Least Squares DPD algorithm, and DPD EIGEN algorithm. After 100 Monte Carlo simulation experiments, the performance comparison results are shown in Figure 6. The SNR varies from $-10 \mathrm{~dB}$ to $15 \mathrm{~dB}$, the signal bandwidth is $B_{\text {signal }}=3 \times 10^{5} \mathrm{~Hz}$, and the signal sampling frequency is $6 \times 10^{7} \mathrm{~Hz}$. There are three receiving base stations with coordinates of $(1000,2000)$, ( $5000,1000)$, and $(10000,0)$, and the coordinate of the source

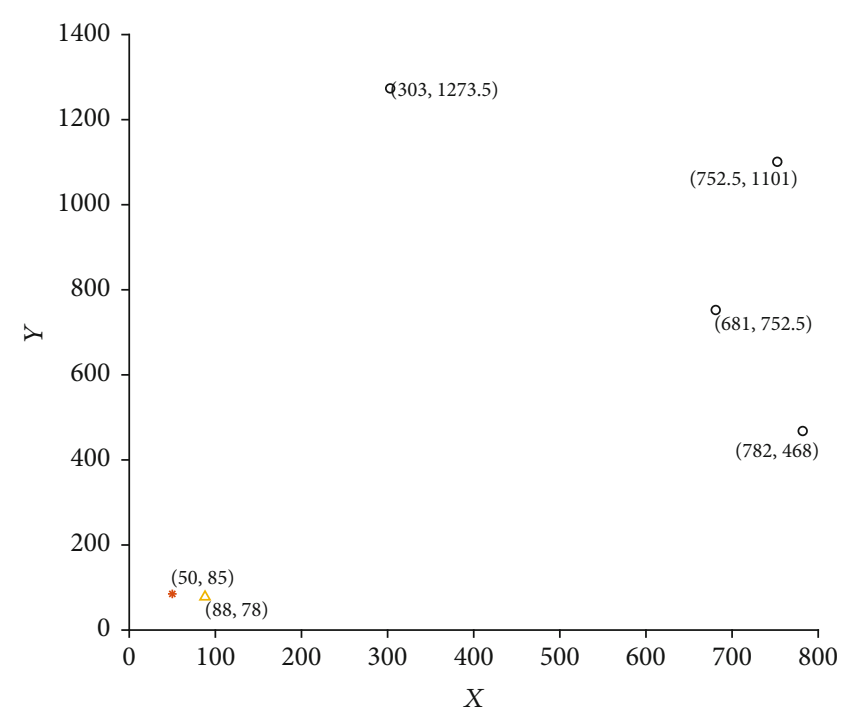

$$
\begin{aligned}
& \text { - Antenna location } \\
& \text { * Signal location } \\
& \triangle \text { Source estimation location }
\end{aligned}
$$

FIgURE 5: Locating result with $\mathrm{SNR}=-5 \mathrm{~dB}$ (under the multipath effect).

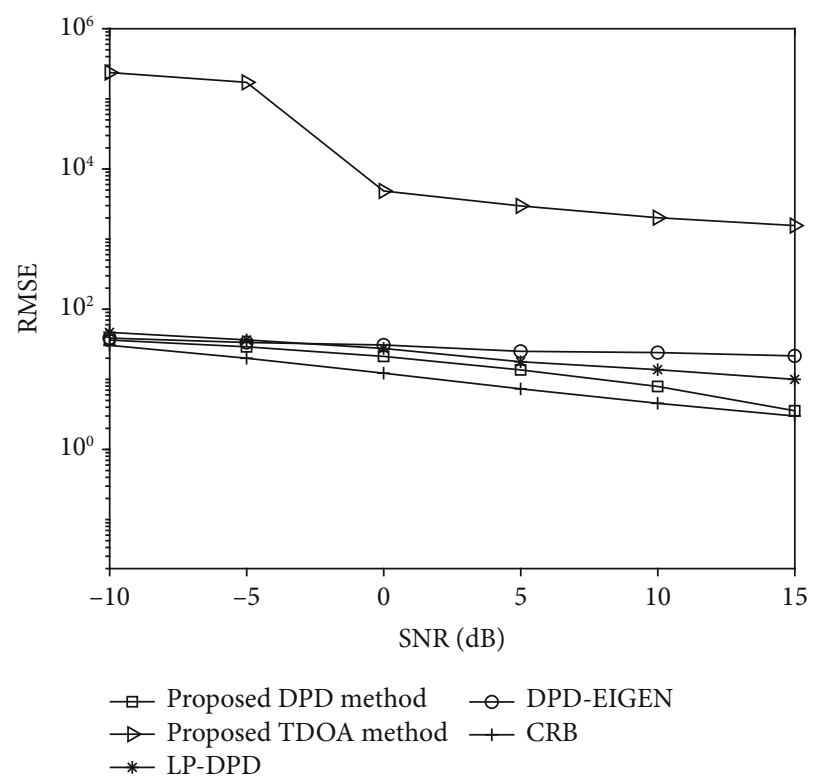

FIgURe 6: Performance comparison versus SNR of the narrowband signal.

is $(135,175)$. It is clear that with the improvement of SNR, the performance of these localization algorithms is getting better. In addition, the performance of the two direct location algorithms is obviously better than that of the improved TDOA algorithm, and the positioning performance of the proposed algorithm is slightly better than that of the traditional direct location algorithm (Least Squares DPD) and DPD EIGEN algorithm. 


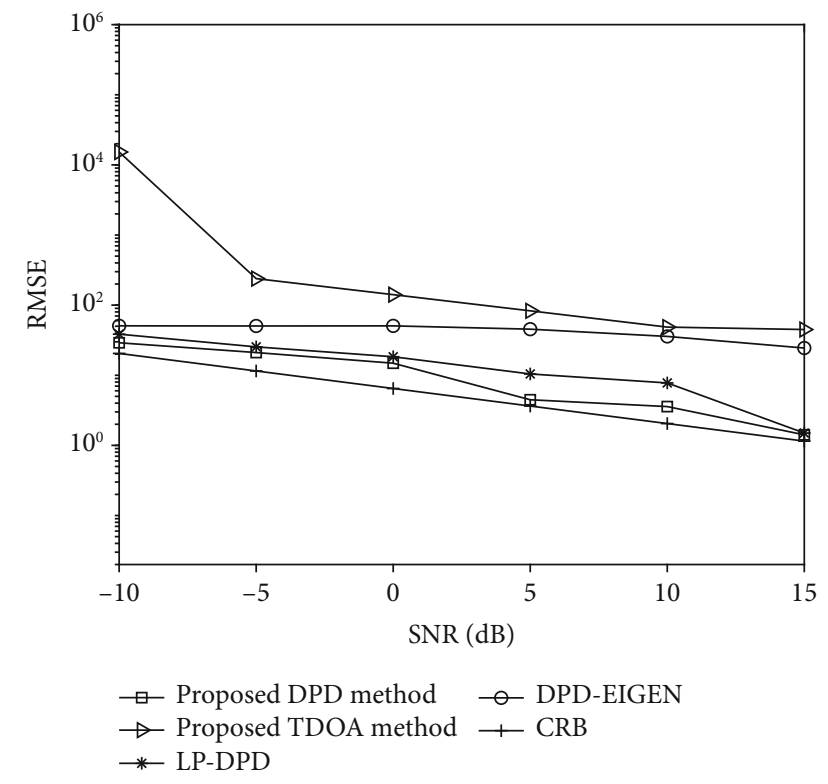

Figure 7: Performance comparison versus SNR of the wideband signal.

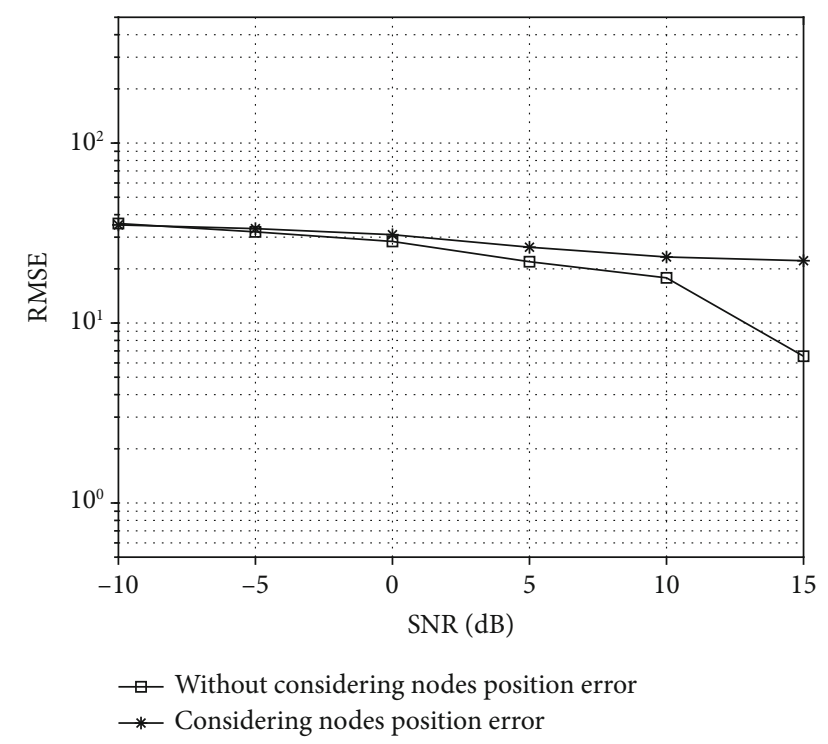

FIGURE 8: Performance comparison when considering node position error.

All else being equal, the signal bandwidth goes from 3 $\times 10^{5} \mathrm{~Hz}$ to $3 \times 10^{6} \mathrm{~Hz}$. Then, the algorithm is compared with the improved TDOA algorithm, Least Squares DPD algorithm, and DPD EIGEN algorithm. After 100 Monte Carlo simulation experiments, the RMSE results are shown in Figure 7. It can be seen that the performance of the proposed algorithm is better than that of the improved TDOA algorithm, Least Squares DPD algorithm, and DPD EIGEN algorithm.

The signal model in this paper is based on the synchronization of all received signals, and the location information of

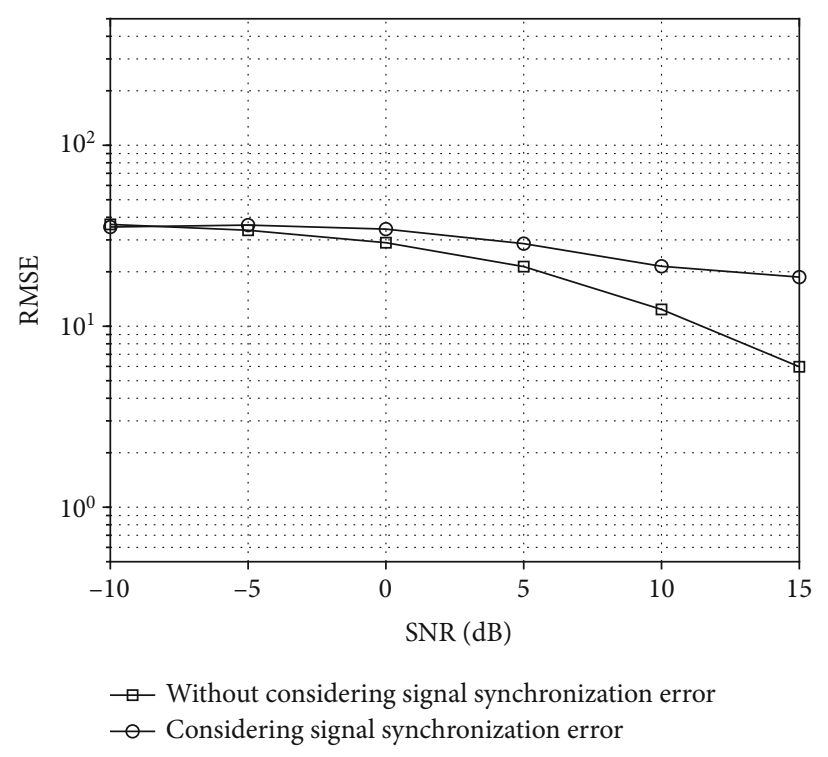

Figure 9: Performance comparison when considering synchronization error.

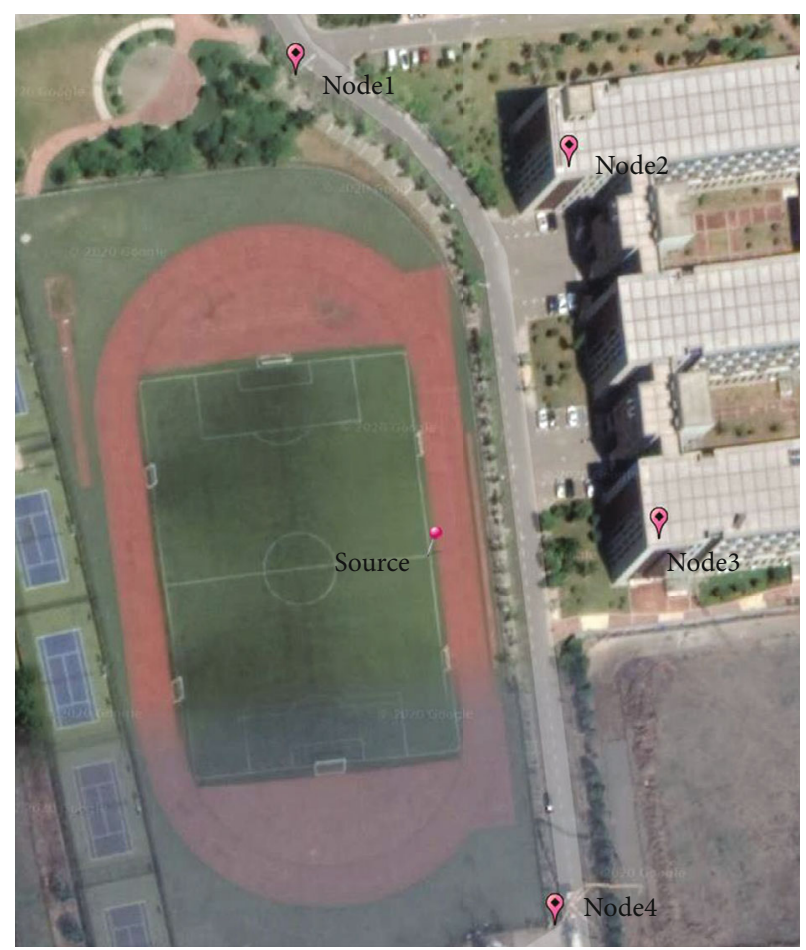

FIgURE 10: Schematic of a distributed positioning system.

each node is accurate. However, in practice, due to various factors, the received signals are not always synchronized, and the position measurement information of each node may also have errors [32, 33]. In order to further analyze the performance of the algorithm, simulation experiments are carried out by considering the position error of the node and the synchronization error of the signal. The experimental results are shown in Figures 8 and 9, respectively. 


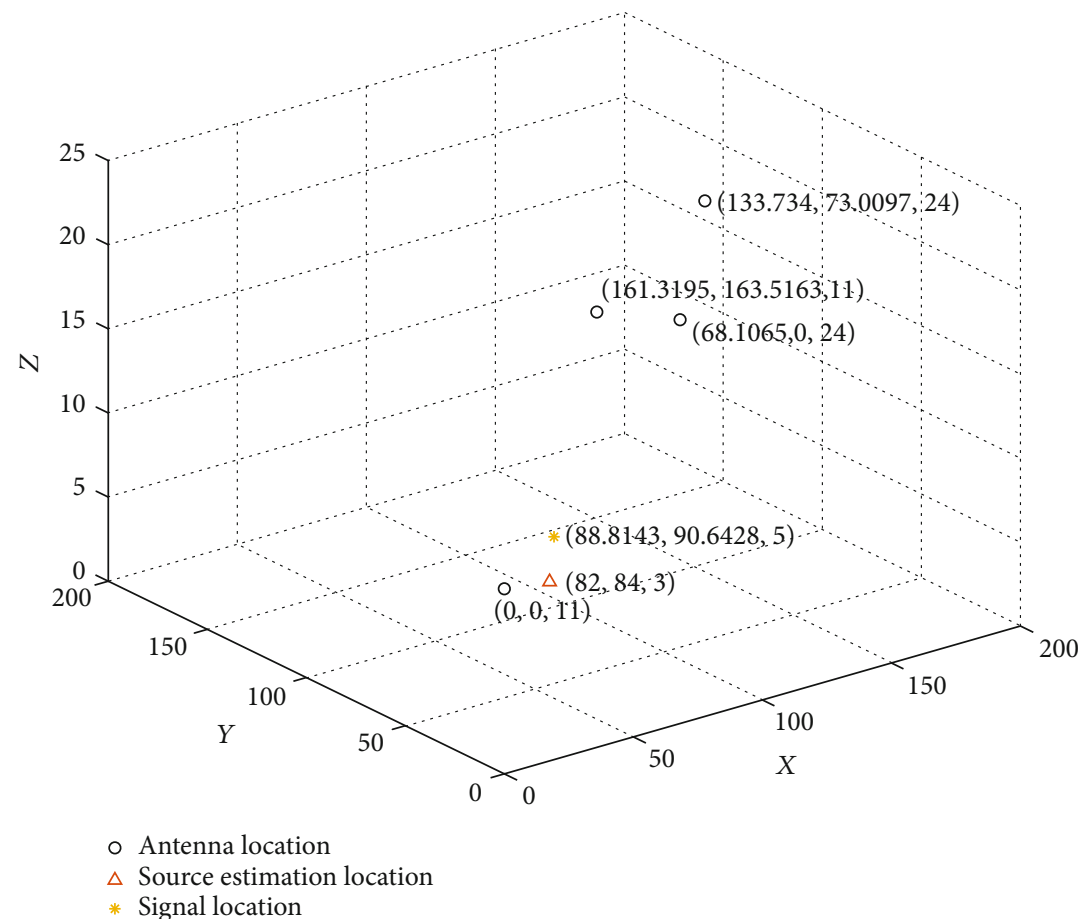

FIgURE 11: Coordinate chart of the positioning result.

After 100 Monte Carlo tests, the results show that the existence of errors has a certain impact on the performance of the algorithm.

5.2. Real Measured Data Processing Results. In order to further verify the effectiveness of the algorithm, the actual measurement data collected in the experiment are processed. As shown in Figure 10, the four nodes in the campus are node 1 , node 2 , node 3 , and node 4 , and the signals are located in the playground area.

In the experiment, the signal source transmits a variety of modulation signals with the power of $5 \mathrm{dBm}, 10 \mathrm{dBm}$, and $15 \mathrm{dBm}$, respectively.

Before processing the data, it is necessary to establish a three-dimensional coordinate space corresponding to the longitude, latitude, and height. In this paper, node 1 in Figure 11 is used as the reference node to establish a three-dimensional coordinate system. Then, the signal with a power of $15 \mathrm{dBm}$ is processed to determine the position of the signal source. The results are shown in Figure 12, from which we can see the relative coordinates of each node, the real location of the source, and the estimated position.

The measured data are processed by the proposed algorithm and the traditional TDOA algorithm, and the positioning results are compared. The data center frequency is $700 \mathrm{MHz}$, and the symbol rate is $40 \mathrm{bit} / \mathrm{s}$. The real coordinates of the source are $(117.6603,49.0477,5)$. The results of the two algorithms are shown in Table 1. It can be seen that the positioning performance of the algorithm proposed in this paper is closer to the actual coordinates compared with the TDOA improved method.

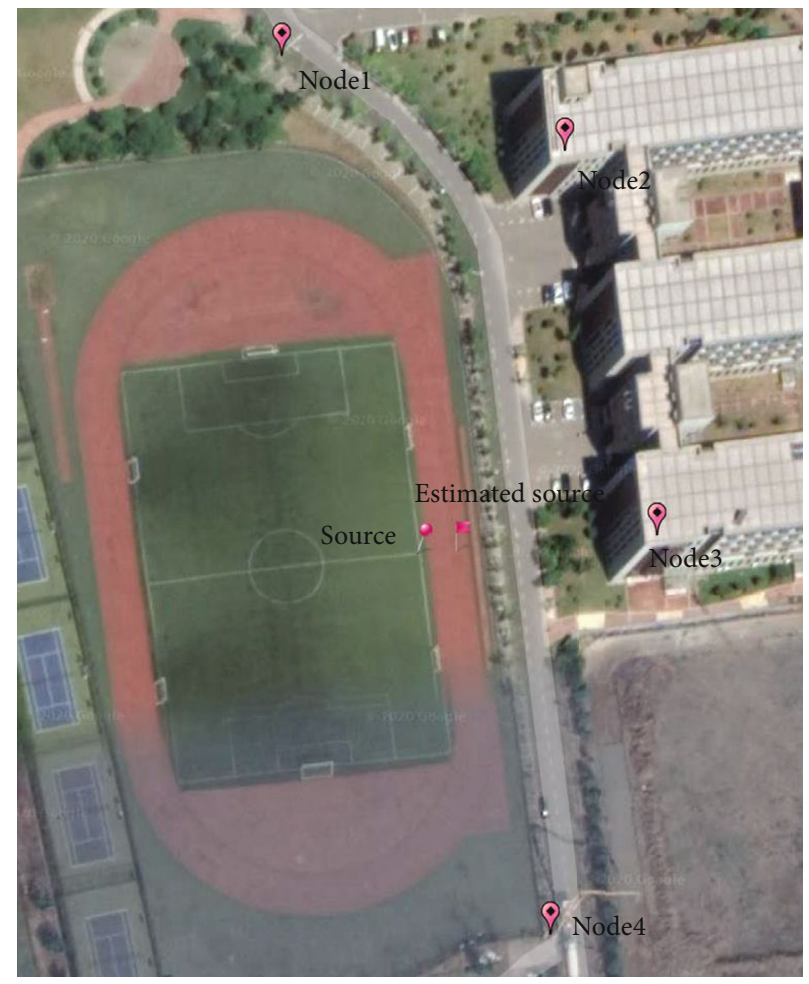

FIGURE 12: Map of positioning results.

Table 2 shows the positioning errors of the two algorithms after processing the three different transmitted power signals. It is clear that the proposed DPD method can achieve more accurate positioning results than the TDOA method. 
TABLE 1: Positioning results of algorithm (unit: $\mathrm{m}$ ).

\begin{tabular}{lccc}
\hline Signal power & $5 \mathrm{dBm}$ & $10 \mathrm{dBm}$ & $15 \mathrm{dBm}$ \\
\hline TDOA & $(105.2617,80.6879,-81.2269)$ & $(108.4732,77.3191,-54.0144)$ & $(96.6336,56.252,-52.1839)$ \\
Proposed DPD & $(131,55,4)$ & $(133,41,6)$ & $(127,49,6)$ \\
\hline
\end{tabular}

TABLE 2: Positioning error (unit: $\mathrm{m}$ ).

\begin{tabular}{lccc}
\hline Signal power & $5 \mathrm{dBm}$ & $10 \mathrm{dBm}$ & $15 \mathrm{dBm}$ \\
\hline TDOA & 92.7 & 66.01 & 63.01 \\
Proposed DPD & 14.6 & 17.4 & 9.4 \\
\hline
\end{tabular}

\section{Conclusion}

DPD algorithm has higher positioning accuracy than the traditional two-step positioning method, but most of the existing direct positioning technologies are aimed at narrowband signals. Based on the phase information of the signal, a correlation direct location algorithm is proposed for both narrowband and wideband signals. The data fusion model of multimonitoring nodes is established, and the simulation of the algorithm is deployed. Simulation results show that the proposed algorithm has higher positioning accuracy and better performance than the traditional TDOA twostep positioning algorithm, LS DPD algorithm, and DPD EIGEN algorithm. In order to further verify the performance of the algorithm, the signal source is used to simulate the interference signal and transmit the signal in the school playground. After collecting data from four nodes, the algorithm proposed in this paper and the improved TDOA algorithm process the measured data. It can be seen that the proposed algorithm has higher positioning accuracy than the TDOA two-step localization algorithm.

\section{Data Availability}

Data are available in the manuscript.

\section{Conflicts of Interest}

The authors declare that they have no conflicts of interest.

\section{Acknowledgments}

This work is supported by the National Science Foundation of China $(61971217,61971218,61631020,61601167)$, the fund of Sonar technology key laboratory (Research on the theory and algorithm of signal processing for twodimensional underwater acoustics coprime array, Range estimation and location technology of passive target via multiple array combination), the Jiangsu Postdoctoral Science Foundation (2020Z013), the China Postdoctoral Science Foundation (2020M681585), and the fund of state key laboratory of complex electromagnetic environment effects on electronics and information system (CEMEE 2021Z0101B).

\section{References}

[1] T. Qin, L. Lin, Z. Lu, and D. Wang, "A ML-based direct localization method for multiple sources with moving arrays," in 2018 18th IEEE International Conference on Communication Technology, Chongqing, China, 2018.

[2] B. Demissie, M. Oispuu, and E. Ruthotto, "Localization of multiple sources with a moving array using supple data fusion," in IEEE llth International Conference on Information Fusion, pp. 1-7, Cologne, Germany, 2008.

[3] Y. Jiexin, W. Ying, and W. Ding, "Direct position determination of multiple noncircular sources with a moving array," Circuits, Systems and Signal Processing, vol. 36, no. 10, pp. 40504076, 2017.

[4] Y. He, J. Li, and X. Zhang, "Adaptive cascaded high-resolution source localization based on collaboration of multi-UAVs," China Communications, vol. 17, no. 4, pp. 165-179, 2020.

[5] W. Yuan, N. Wu, B. Etzlinger, Y. Li, C. Yan, and L. Hanzo, "Expectation-maximization-based passive localization relying on asynchronous receivers: centralized versus distributed implementations," IEEE Transactions on Communications, vol. 67, no. 1, pp. 668-681, 2019.

[6] L. Wan, K. Liu, Y.-C. Liang, and T. Zhu, "DOA and polarization estimation for non-circular signals in 3-D millimeter wave polarized massive MIMO systems," IEEE Transactions on Wireless Communications, vol. 20, no. 5, pp. 3152-3167, 2021.

[7] L. Wan, Y. Sun, L. Sun, Z. Ning, and J. J. P. C. Rodrigues, "Deep learning based autonomous vehicle super resolution DOA estimation for safety driving," IEEE Transactions on Intelligent Transportation Systems, pp. 1-15, 2020.

[8] T. Tirer and A. J. Weiss, "Performance analysis of a highresolution direct position determination method," IEEE Transactions on Signal Processing, vol. 65, no. 3, pp. 544-554, 2017.

[9] F. Qi, Q. Changwen, and Z. Qiang, "Direct position determination using asynchronous observation of multiple moving sensors," Journal of Electronics \& Information Technology, vol. 39, no. 2, pp. 417-422, 2017.

[10] A. Weiss, "Direct position determination of narrowband radio frequency transmitters," IEEE Signal Processing Letters, vol. 11, no. 5, pp. 513-516, 2004.

[11] T. Tirer and A. J. Weiss, "High resolution direct position determination of radio frequency sources," IEEE Signal Processing Letters, vol. 23, no. 2, pp. 192-196, 2016.

[12] W. Daming, R. Yanqing, L. Zhiyu, B. Bin, and C. Weijia, "Array-based direct position determination method fusing Doppler frequency shift information," Journal of Electronics \& Information Technology, vol. 40, no. 5, pp. 1219-1225, 2018.

[13] J. Bosse, A. Ferreol, and P. Larzabal, "Performance analysis of passive localization strategies: direct one step approach versus 2 steps approach," in 2011 IEEE Statistical Signal Processing Workshop (SSP), pp. 701-704, Nice, France, 2011. 
[14] L. U. Zhiyu, B. A. Bin, W. A. N. G. Jianhui, L. I. Wenchao, and W. A. N. G. Daming, "A direct position determination method with combined TDOA and FDOA based on particle filter," Chinese Journal of Aeronautics, vol. 31, no. 1, pp. 161-168, 2018.

[15] L. Zhou, W. Zhu, J. Luo, and H. Kong, "Direct positioning maximum likelihood estimator using TDOA and FDOA for coherent short-pulse radar," IET Radar, Sonar \& Navigation, vol. 11, no. 10, pp. 1505-1511, 2017.

[16] Y. Wang and Y. Wu, "An improved direct position determination algorithm with combined time delay and Doppler," Journal of Xi'an Jiaotong University, vol. 49, no. 4, pp. 123-129, 2015.

[17] E. Tzore and A. J. Weiss, "Expectation-maximization algorithm for direct position determination," Signal Processing, vol. 133, pp. 32-39, 2017.

[18] G. Wang, C. Gao, S. G. Razul, and C. M. S. See, "A new direct position determination algorithm using multiple arrays," in 2018 IEEE 23rd International Conference on Digital Signal Processing (DSP), pp. 1-5, Shanghai, China, 2018.

[19] Y. Jiexin, D. Wang, Y. Wu, and L. Ruirui, "Direct localization of multiple stationary narrowband sources based on angle and Doppler," IEEE Communications Letters, vol. 21, no. 12, pp. 2630-2633, 2017.

[20] O. Bialer, D. Raphaeli, and A. J. Weiss, "Maximum-likelihood direct position estimation in dense multipath," IEEE Transactions on Vehicular Technology, vol. 62, no. 5, pp. 2069-2079, 2013.

[21] J. S. Picard and A. J. Weiss, "Direct position determination sensitivity to NLOS propagation effects on Doppler-shift," IEEE Transactions on Signal Processing, vol. 67, no. 14, pp. 3870-3881, 2019.

[22] A. Fascista, A. Coluccia, H. Wymeersch, and G. Seco-Granados, "Millimeter-wave downlink positioning with a singleantenna receiver," IEEE Transactions on Wireless Communications, vol. 18, no. 9, pp. 4479-4490, 2019.

[23] A. Fascista, A. Coluccia, and G. Ricci, "A pseudo maximum likelihood approach to position estimation in dynamic multipath environments," Signal Processing, vol. 181, article 107907, 2020.

[24] A. J. Weiss, "Direct geolocation of wideband emitters based on delay and Doppler," IEEE Transactions on Signal Processing, vol. 59, no. 6, pp. 2513-2521, 2011.

[25] N. Vankayalapati, S. Kay, and Q. Ding, "TDOA based direct positioning maximum likelihood estimator and the CramerRao bound," IEEE Transactions on Aerospace and Electronic Systems, vol. 50, no. 3, pp. 1616-1635, 2014.

[26] I. P. Pokrajac, D. Vucic, and P. Okiljevic, "Direct position determination of wideband signals: coherent and noncoherent approach," in 2013 11th International Conference on Telecommunications in Modern Satellite, Cable and Broadcasting Services (TELSIKS), pp. 77-80, Nis, Serbia, 2013.

[27] D. Čabrić and M. Erić, "Spatio-temporal spectrum sensing using distributed antenna systems and direct localization methods," in Proceedings of the 2012 IEEE International Symposium on Antennas and Propagation, pp. 1-2, Chicago, IL, USA, 2012.

[28] A. T. Ott, M. Shalaby, U. Siart et al., "System simulation of a localization system based on power level detection with distributed antennas," in Proceedings of the 5th European Conference on Antennas and Propagation (EUCAP), pp. 19-23, Rome, Italy, 2011.
[29] M. Zhou, Y. Wang, Y. Liu, and Z. Tian, "An informationtheoretic view of WLAN localization error bound in GPSdenied environment," IEEE Transactions on Vehicular Technology, vol. 68, no. 4, pp. 4089-4093, 2019.

[30] L. Wan, L. Sun, K. Liu, X. Wang, Q. Lin, and T. Zhu, “Autonomous vehicle source enumeration exploiting non-cooperative UAV in software defined internet of vehicles," IEEE Transactions on Intelligent Transportation Systems, pp. 1-13, 2020.

[31] B. Demissie, M. Oispuu, and E. Ruthotto, "Localization of multiple sources with a moving array using subspace data fusion," in 2008 11th International Conference on Information Fusion, pp. 1-7, Cologne, Germany, 2008.

[32] Y. Xiong, N. Wu, Y. Shen, and M. Z. Win, "Cooperative network synchronization: asymptotic analysis," IEEE Transactions on Signal Processing, vol. 66, no. 3, pp. 757-772, 2018.

[33] W. Yuan, N. Wu, Q. Guo, X. Huang, Y. Li, and L. Hanzo, "TOA-based passive localization constructed over factor graphs: a unified framework," IEEE Transactions on Communications, vol. 67, no. 10, pp. 6952-6965, 2019. 